\title{
Exercício Físico e Teste de Caminhada de 6-min na Doença Arterial Obstrutiva de Membros Inferiores
}

\author{
Exercise and Six-Minute Walk Test in Lower Extremity Occlusive Peripheral Arterial Disease
}

\author{
Tales de Carvalho $0^{1,2}$ \\ Clínica de Prevenção e Reabilitação Cardiosport, ${ }^{1}$ Florianópolis, SC - Brasil \\ Universidade do Estado de Santa Catarina (UDESC-SC), ${ }^{2}$ Florianópolis, SC - Brasil \\ Minieditorial referente ao artigo: Intensidade de Exercício durante o Teste de Caminhada de 6 Minutos em Pacientes com Doença Arterial Periférica
}

$\mathrm{O}$ artigo "Intensidade de Exercício durante o Teste de Caminhada de 6 Minutos em Pacientes com Doença Arterial Periférica", ${ }^{1}$ com originalidade, produz informações de grande utilidade prática, a serem consideradas no diagnóstico, prognóstico e principalmente na avaliação funcional, que possibilitam um aprimoramento da prescrição de exercícios físicos no tratamento clínico da doença. O estudo foi realizado com o objetivo de determinar em pacientes sintomáticos com Doença Arterial Obstrutiva (DAO) de Membros Inferiores (MMII) se a caminhada no solo permite a detecção do primeiro limiar ventilatório, também denominado limiar anaeróbio (LA). O LA é um marcador reconhecido de intensidade do exercício, útil para a delimitação da zona considerada ideal para o treinamento físico visando o aprimoramento cardiorrespiratório. ${ }^{2}$

A DAO de MMII é um grande problema de saúde pública, que de acordo com o relatório global de epidemiologia, acometia 202 milhões de indivíduos em 2010, crescendo $22 \%$ até 2015, quando atingiu 237 milhões de indivíduos. ${ }^{3}$ A associação da DAO com grandes eventos cardiovasculares (MACE) está bem documentada; em seu estágio mais grave, com isquemia crítica, apresenta elevado risco de eventos cardiovasculares, amputação de MMII e morte, ${ }^{4}$ associando-se a níveis elevados de troponina cardíaca e peptídeo natriurético cerebral pro-N-terminal (NT proBNP). ${ }^{5}$

Há forte suspeita de DAO de MMII quando ocorre dor em MMII ao esforço sem aparente etiologia ortopédica e o índice tornozelo-braquial (ITB) é menor que 0,90 em repouso. Os testes de esforço realizados com caminhadas devem ser realizados para auxiliar no diagnóstico, especialmente quando o ITB for maior que 0,91, sendo úteis também para classificação funcional e prescrição de exercícios. ${ }^{6}$ Os testes de campo possibilitam o diagnóstico de claudicação intermitente, com determinação das distâncias percorridas para o início da sintomatologia (claudicação inicial) e para o surgimento da

\section{Palavras-chave}

Doença Arterial Periférica; Claudicação Intermitente; Limiar Anaeróbio; Exercício; Teste de Caminhada; Atividades Físicas da Vida Diária.

Correspondência: Tales de Carvalho •

Avenida Jornalista Rubens de Arruda Ramos, 2354, apto 201. CEP: 88015-705, Florianópolis, SC - Brasil

E-mail: tales@cardiol.br, talescarvalho@icloud.com

DOI: https://doi.org/10.36660/abc.20200068 máxima limitação funcional (claudicação absoluta), quando o indivíduo é obrigado a interromper a caminhada. Nos testes em esteira tem sido proposta a inclusão da medida do ITB em repouso e após exercício. A presença da doença é fortemente sugerida quando ocorre redução do ITB pós-exercício superior a $20 \%$ e a $30 \mathrm{mmHg}$ em relação ao observado ao repouso. ${ }^{7} \mathrm{O}$ ITB em repouso, que tem sido amplamente utilizado na prática clínica, pode com certa frequência produzir um resultado falso negativo, exigindo atenção a grupos com artérias não compressíveis. No estudo de Tóth-Vajna et al., ${ }^{8}$ quase um quarto dos indivíduos com diagnóstico apresentou artéria não compressível ou foi considerado sintomático com ITB negativo. Portanto, se houver suspeita clínica de DAO dos MMII, apesar dos valores normais do ITB, uma avaliação mais aprofundada deve ser considerada.

Em pacientes sintomáticos os exercícios físicos têm potencial para influir na morbidade e mortalidade, proporcionando redução dos sintomas, melhora da qualidade de vida e aumento da distância máxima caminhada, devendo obrigatoriamente integrar o tratamento otimizado., ${ }^{6,7}$ Portanto, todos os pacientes com claudicação intermitente devem receber tratamento clínico otimizado, ou seja, uma associação de mudanças de estilo de vida com tratamento farmacológico, tendo em vista as evidências que demonstram redução de eventos cardiovasculares e melhora de desfechos relacionados aos MMII. ${ }^{9}$

O treinamento físico tem se mostrado seguro, sendo os exercícios realizados por meio de caminhadas com indução do sintoma de claudicação a melhor opção. ${ }^{6,7}$ Mas, quando a caminhada não puder ser realizada, outros tipos de exercícios, como ciclismo, exercícios resistidos e ergômetro de membros superiores, têm se mostrado efetivos. ${ }^{6,7}$ Cabe ainda ressaltar que exercícios físicos não podem ser realizados por pacientes com isquemia crítica, mas devem ser considerados o mais breve possível após tratamento intervencionista realizado com sucesso. ${ }^{10,11}$

Muitos ensaios clínicos demonstraram consistentemente que o exercício supervisionado em esteira melhora o desempenho da marcha em pessoas com DAO de MMII. A metanálise de Fakhry et al., ${ }^{12}$ que avaliou 1054 indivíduos em 25 estudos, concluiu que esse tipo de exercício proporcionou em média aumento de 180 metros na distância máxima de caminhada na esteira e de 128 metros na distância caminhada sem dor. ${ }^{12}$

Três ensaios clínicos randomizados, que avaliaram um total de 493 pacientes com DAO de MMII, demonstraram que as intervenções com exercícios baseados em domicílio, 
que incorporaram técnicas de mudança comportamental, aumentaram a capacidade para caminhar, sendo superiores em proporcionar ganho no desempenho no teste de caminhada de seis minutos na comparação com os exercícios supervisionados realizados em esteira. ${ }^{13,14}$ Ou seja, enquanto programas de exercícios supervisionados em esteira são superiores em aumentar o desempenho da caminhada na esteira, os programas de exercícios baseados em domicílio são superiores para melhorar o desempenho em caminhada no solo, algo mais bem relacionado às atividades da vida diária ${ }^{14}$

Embora em estudos mais recentes os exercícios de caminhada no solo baseados em domicílio tenham se mostrado mais eficazes para o desempenho nas atividades da vida diária, sendo mais indicados do que os exercícios supervisionados em esteira, ${ }^{14}$ os pequenos estudos iniciais mostraram pouco ou nenhum benefício, justificando que nas Diretrizes de Prática Clínica do American College of Cardiology/American Heart Association de 2006 constasse equivocadamente "não haver evidências para aconselhar os pacientes a irem para casa caminhar". Porém, desde 2011 foram publicados ensaios clínicos bem sucedidos que tiveram como intervenção exercícios baseados em domicílio e incluíram substancialmente mais do que conselhos para "ir para casa e caminhar", solicitando aos pacientes para que estabelecessem metas de exercícios e monitorassem suas atividades físicas, ocasionando a mudança do enfoque. Mesmo com componentes de intervenção comportamental, o exercício baseado em domicílio requer menos recursos e menores custos que o exercício supervisionado em esteira, sendo mais acessíveis e provavelmente mais aceitáveis para muitos pacientes, o que poderia favorecer a uma maior adesão ao tratamento. ${ }^{13}$

$\mathrm{Na}$ avaliação funcional de pacientes com DAO de MMII, o teste de caminhada de seis minutos vem ganhando espaço, como uma medida validada, mais bem relacionada aos níveis de atividade física que o teste em esteira, sem estar associado a um efeito de aprendizado quando testes repetidos são realizados. ${ }^{14} \mathrm{Em}$ pacientes com DAO de MMII, em comparação aos testes realizados em esteira, as alterações no desempenho observadas na caminhada de seis minutos têm sido melhor associadas a desfechos como mortalidade e perda de mobilidade. ${ }^{14}$

No artigo "Intensidade de Exercício durante o Teste de Caminhada de 6 Minutos em Pacientes com Doença Arterial Periférica", ${ }^{1}$ o LA foi alcançado no teste de caminhada de 6 minutos em $60 \%$ dos indivíduos. O consumo pico de oxigênio obtido durante o teste em esteira, assim como o ITB, foram maiores nos pacientes que não alcançaram o LA durante o teste de caminhada de 6 minutos. O teste de caminhada de 6 minutos foi mais difícil para mulheres e pacientes com baixa aptidão cardiorrespiratória, revelando maior intensidade relativa de esforço de exercício para esses pacientes. Isso é algo relevante na prática, pois visando à melhora da função cardiovascular, recomenda-se que o treinamento físico seja realizado acima do LA. ${ }^{15}$ Portanto, o artigo mostra que existe fundamentação para a utilização de caminhada no solo como modo de exercício preferencial principalmente para mulheres e pacientes com baixa aptidão cardiorrespiratória. Para homens e pacientes com maior aptidão cardiorrespiratória devem ser considerados exercícios mais intensos, em cicloergômetros, ergômetros elípticos ou mesmo esteiras, visando atingir o LA e, consequentemente, melhorar aptidão física.

Por fim, vale mais uma vez ressaltar que o artigo "Exercise Intensity During 6-min Test in Patients with Peripheral Artery Disease" apresenta originais e interessantes resultados, mas sendo um estudo observacional unicêntrico, com pequeno número de indivíduos, obviamente, apresenta limitações que impedem fortes conclusões. Portanto, as importantes informações aplicáveis à prática clínica que constam nos resultados do estudo publicado nesse artigo merecem ser consideradas como temas de futuras pesquisas.

\section{Referências}

1. Farah BQ, Ritti-Dias RM, Montgomery P, Cucato GG, Gardner A. Exercise Intensity during 6-Minute Walk Test in Patients with Peripheral Artery Disease. Arq Bras Cardiol. 2020; 114(3):486-492

2. Neder JA, Nery LE. Teste de Exercício Cardiopulmonar. J Pneumol. 2002; 28(Supl 3): 166-206

3. Song P, Rudan D, Zhu Y, Fowkes FJI, Rahimi K, Fowkes FGR, et al. Global, regional, and national prevalence and risk factors for peripheral artery disease in 2015: an updated systematic review and analysis. Lancet Glob Health. 2019;7(8): e1020-e1030.

4. Beckman JA, Duncan MS, Damrauer SM, Wells QS, BarnettJV, Wasserman $\mathrm{DH}$, et al. Microvascular disease, peripheral artery disease, and amputation. Circulation. 2019;140(6):449-58.

5. Matsushita K, Kwak L, Yang C, Pang Y, Ballew SH, Sang Y, et al. Highsensitivity cardiac troponin and natriuretic peptide with risk of lower extremity peripheral artery disease: the Atherosclerosis Risk in Communities (ARIC) Study. Eur Heart J. 2018;39(25):2412-9.
6. Aboyans V, Ricco JB, Bartelink MEL, Bjorck M, Brodmann M, Cohnert T, et al; ESC Scientific Document Group. 2017 ESC Guidelines on the Diagnosis and Treatment of Peripheral Arterial Diseases, in collaboration with the European Society for Vascular Surgery (ESVS): document covering atherosclerotic disease of extracranial carotid and vertebral, mesenteric, renal, upper and lower extremity arteries Endorsed by: the European Stroke Organization (ESO) The Task Force for the Diagnosis and Treatment of Peripheral Arterial Diseases of the European Society of Cardiology (ESC) and of the European Society for Vascular Surgery (ESVS). Eur Heart J. 2018;39(9):763-816.

7. Gerhard-Herman MD, Gornik HL, Barrett C, Barshes NR, Corriere MA Drachman DE, et al. 2016 AHA/ACC Guideline on the Management of Patients With Lower Extremity Peripheral Artery Disease: Executive Summary: A Report of the American College of Cardiology/American Heart Association Task Force on Clinical Practice Guidelines. Circulation. 2017;135(12):e686-e725

8. Tóth-Vajna Z, Tóth-Vajna G, Gombos Z, Szilágyi B, Járai Z, Berczeli M Sótonyi P. Screening of peripheral arterial disease in primary health care. Vascular Health Risk Manag. 2019 Aug 20;15:355-63. 


\section{Minieditorial}

9. Arya S, Khakharia A, Binney ZO, DeMartino RR, Brewster LP, Goodney PP, et al. Association of Statin Dose With Amputation and Survival in Patients With Peripheral Artery Disease. Circulation. 2018;137(14):1435-46.

10. Klaphake S, Buettner S, Ultee KH, van Rijn MJ, Hoeks SE, Verhagen HJ. Combination of endovascular revascularization and supervised exercise therapy for intermittent claudication: a systematic review and meta-analysis. J Cardiovasc Surg (Torino). 2018;59(2):150-7.

11. Saratzis A, Paraskevopoulos I, Patel S, Donati T, Biasi L, Diamantopoulos A, et al. Supervised Exercise Therapy and Revascularization for Intermittent Claudication: Network Meta-Analysis of Randomized Controlled Trials. JACC Cardiovasc Interv. 2019;12(12):1125-36.
12. Fakhry F, van de Luijtgaarden KM, Bax L, den Hoed PT, Hunink MG, Rouwet EV, Spronk S. Supervised walking therapy in patients with intermittent claudication. J Vasc Surg. 2012;56(4):1132-42.

13. McDermott MM, Polonsky T. Home-based exercise: a therapeutic option for peripheral artery disease. Circulation. 2016; 134(16):1127-9.

14. McDermott MM Guralnik JM, Criqui MH, Liu K, Kibbe MR, Ferrucci L. Six-minute walk is a better outcome measure than treadmill walking tests in therapeutic trials of patients with peripheral artery disease. Circulation. 2014; 130(1):61-8.

15. Temfemo A, Chlif M, Mandengue SH, Lelard T, Choquet D, Ahmaidi S. Is there a beneficial effect difference between age, gender, and different cardiac pathology groups of exercise training at ventilatory threshold in cardiac patients? Cardiol J. 2011;18(6):632-8.

Este é um artigo de acesso aberto distribuído sob os termos da licença de atribuição pelo Creative Commons 